\section{How a database of nuclear databases could help the effort to combat trafficking}

SIR - We welcome the views expressed in the Commentaryby M. May, J. Davis and R. Jeanloz on source attribution of nudear materials ("Preparing for the worst", Nature $443,907-908 ; 2006$ ), in particular the suggestion to establish an international databank of such material to support the interpretation of nuclear forensics analyses.

We would like to comment on a few points, in particular those where reference was made to the Institute for Transuranium Elements (ITU) in Karlsruhe, part of the European Commission's Joint Research Centre.

The ITU has been involved since the early 1990s in the analysis of seized nuclear materials from cases of illicit trafficking. Its database, mentioned in the Commentary, contains materials from several western European and Russian fuel manufacturers, and was established in collaboration with the Bochvar Institute in Moscow. For reasons of commercial sensitivity, its content is largely confidential. Nevertheless, we assess information requests on a case-by-case basis.

Most nudear or radioactive material has been seized in quantities too small for the production of a nuclear device. However, it is conceivable that small quantities could be used by terrorists for a radiological dispersal device, or 'dirty bomb', which would also require source attribution.

As your Commentary mentions, the Nuclear Smuggling International Technical Working Group discusses scientific and technical aspects of nuclear forensics analyses. Its 2006 meeting discussed various options for information sharing, including the creation of a 'super' database containing information about other organizations' databases. This decentralized approach may be easier to implement than a single centralized database. Non-nuclear states also need to be involved in this effort.

We agree that incidents involving nuclear material call for a comprehensive response, which relies on an organizational and technical infrastructure. This includes 'crime scene' management to preserve forensic evidence - nudear as well as traditional and maintain the chain of custody.

Credible nuclear forensics is based on representative samples and on high-quality analyses carried out in specialized multidisciplinary laboratories. Some parameters (such as age, intended use and production mode) are self-explanatory. But others (such as impurities and pellet dimensions) can best be understood by comparison to references, which calls for information on a wide variety of materials or the ability to pose queries in databases. Source attribution is essential in the fight against illicit trafficking and in the prevention of malicious acts. Klaus Luetzenkirchen, Klaus Mayer Institute for Transuranium Elements, Joint Research Centre, European Commission, PO Box 2340, Karlsruhe 76125, Germany

\section{Time to give due weight to the 'carbon footprint' issue}

SIR - The media are increasingly using the term 'carbon footprint' in articles about the need to mitigate climate change by reducing our carbon dioxide emissions. Footprints are spatial indicators, measured in hectares or square metres. The property that is often referred to as a carbon footprint is actually a 'carbon weight' of kilograms or tonnes per person or activity.

To improve public understanding of the issues surrounding climate change, it is necessary to be precise. Other 'footprints', such as the ecological or environmental footprint, convert resource consumption and waste production into spatial units. The term 'ecological footprint' was coined by William E. Rees, a planner at the University of British Columbia - who had previously used the term 'appropriated carrying capacity' - after a computer delivery man told him that the new machine, which took up less space than his old model, had a 'smaller footprint'.

As well as the media, many government agencies and environmental groups now use the expression 'carbon footprint'. Those who favour precision in such matters should perhaps campaign for it to be called 'carbon weight', or some similar term. That would avoid lasting confusion. Losing weight might even take on a whole new meaning. Geoffrey Hammond International Centre for the Environment, and Department of Mechanical Engineering University of Bath, Bath BA2 7AY, UK

\section{Moon and Venus as worthy of exploration as Mars}

SIR - While joining in your celebration of achievements by Mars Global Surveyor and remaining Mars spacecraft (Nature 444, 519 and 526-527; 2006), I disagree on priorities. The Editorial commends "the idea of a continuous presence" on Mars, with "various spacecraft calibrating and complementing each other's results". Yet the related News story seems to accept the view that "other missions might be easier to finish, such as Venus Express - there's a limit to how much useful data can be gathered by continuing to orbit Venus with the same instruments".

According to this 'science per dollar' principle, if continuous and intensive exploration is appropriate for Mars, then it is also appropriate for Venus, which is no more costly to reach and features a dynamically varying atmosphere and surface that have evolved in an intriguingly different way from Earth. The same principle also applies to the Moon. Although not dynamically changing, itholds valuable records of the early Earth's environment, and is reachable in a fraction of the time it takes to reach the planets.

Let us hope that the new US Congress and its international counterparts take the best ideas from all sources and assemble a balanced programme of lunar and planetary exploration for the coming decade.

Curt Covey

1010 LynnStreet, Livermore,

California 94550, USA

\section{Need to speak English puts burden on Asian scientists}

SIR - Masao Ito and Torsten Wiesel point out in Correspondence that factors such as non-standard design or a lack of English on homepages make it harder for the Human Frontier Science Program to identify potential project collaborators or reviewers ( ${ }^{\circ}$ Cultural differences reduce Japanese researchers' visibility on the Web" Nature $444,817,2006)$. However, it is surely not the case that only those whose details are easily found through Web-based searching will be able to compete effectively. Inhomogeneities in the ability of scientists and institutions to perform internationally competitive research have been with us for many decades, and I doubt whether use of the Web has perturbed this pattern to any substantial extent.

I would also dispute Ito and Wiesel's contention that perfecting English-language homepages is a 'simple remedy.' Having to communicate in English to survive, let alone flourish, in the international scientific arena places enormous additional burdens on scientists throughout Asia. Working in a young science-based university in Japan, I see these obstacles being confronted every day. Reading the literature, writing manuscripts, giving oral presentations at conferences - all of these are difficult enough for native speakers of English, but they are fearsome tasks for Asian scientists. In my experience, most English-speaking scientists are blissfully unaware of their magnitude.

The brightest minds in Asian science, adept not only as scientists but also as linguists, will easily possess the wherewithal to create homepages that do them justice. It is with their equally scientifically gifted but less linguistically capable colleagues that we should be concerned. lan Smith

Shikanodai-Nishi 3-6-10, lloma, Nara 630-0114, Japan 